\title{
Comparative Evaluation of Performance of Qualitative and Semiquantitative Slide Widal Test versus Quantitative Tube Widal Test in Diagnosing Enteric Fever
}

\author{
N. Keerthana ${ }^{1}$, R. Someshwaran ${ }^{2}$ and A. Dhanasekaran ${ }^{2 *}$ \\ ${ }^{1}$ Karpagam Faculty of Medical Sciences and Research, Othakkalmandapam, \\ Coimbatore-641032, Tamil Nadu, India \\ ${ }^{2}$ Department of Microbiology, Karpagam Faculty of Medical Sciences and Research, \\ Othakkalmandapam, Coimbatore-641032, Tamil Nadu, India \\ *Corresponding author
}

\section{A B S T R A C T}

Keywords

Salmonella typhi,

Typhoid fever,

Septicemia, Infection

control, Agglutination,

Serological test, Blood

culture, Sensitivity

Article Info

Accepted:

12 October 2018

Available Online:

10 November 2018
Enteric fever still remains as an important cause for morbidity and mortality throughout the world and the causative organism for Typhoid and paratyphoid fever are Salmonella typhi and Salmonella paratyphi $A, B$ and $C$ respectively. Objective of the study is to compare and evaluate the performance of Qualitative and semiquantitative Slide Widal test and Quantitative Tube Widal test for diagnosis of enteric fever. A cross sectional study was undertaken and about 400paired serum samples were obtained from the patients and were subjected to both slide and tube widal tests. The results were noted for further statistical analysis. Prevalence of Enteric fever by tube agglutination and slide agglutination tests were found to be $23 \%$ (92 out of 400 cases) and 13\% (52 out of 400 cases) respectively. Though blood culture remains gold standard, its sensitivity is high only during the first week of fever. So, Widal test is the preferred test used by most clinicians especially during second week of fever. The sensitivity and specificity of tube widal test is much remarkable and so it can definitely be used as a diagnostic test. Slide Widal test can be used as supplementary test to Tube Widal test so as to fetch the results earlier and to facilitate early and appropriate management.

\section{Introduction}

Enteric fever has been an important cause of morbidity and mortality throughout the world for centuries. The causative organism for Enteric fever viz., Typhoid and paratyphoid fever are Salmonella typhi and Salmonella paratyphi $A, B$ and $C$ respectively (John et al., 2016). Though the overall incidence and prevalence has decreased significantly, infection still remains endemic in most of the developing countries. Global prevalence of Typhoid and paratyphoid fever was $10-11.7 \%$ and 2.2-2.5\% in 2016, respectively. Prevalence of Typhoid fever and paratyphoid fever in India was $31.5-36 \%$ and $8-9 \%$ in 2016, respectively (Dandona et al., 2017). Poor sanitation largely contributes to the 
persistence of infection. Improvement in hygiene and timely diagnosis coupled with appropriate treatment ensuring complete cure of cases and carriers remains the keystone in controlling the infection. Culturing the causative bacteria from various body fluids like blood, bone marrow, stool, bile and urine and detection of anti-Salmonella antibodies through serology remains the most common diagnostic tests. Isolation of Salmonella species using Wilson and Blair medium (selective media), which remains the gold standard investigation till date, is greatly influenced by the time duration of illness and appropriateness of the sample chosen for culture (Olopoenia and King, 2000; Mengist and Tilahun, 2017).

Yield of a positive blood culture is very high during the first week of the illness, followed by a marked decrease in its sensitivity. The habit of patients getting started on few doses of antibiotics before the culture has made the investigation lesser sensitive. Hence serological test-Tube Widal test is the most preferred choice by the physicians. It is found more useful in case of culture negative but clinically suspected cases. Widal was first introduced by Georges Fernand Widal in 1896. This test has evolved since then. With the introduction of commercial kits, it has becoming one of the easily available and performable tests.

The quicker result issued in one day, compared to culture (may need 7 days) makes it more useful investigation to decide on the treatment. Recent advancements like Slide Widal test have made the procedure much simpler and very rapid which offers results in one minute. The literature available on its diagnostic ability is however scanty (Olopoenia and King, 2000; Keddy et al., 2011). Hence this study is undertaken to see if the performance of Slide widal is as efficacious as tube widal test.

\section{Study rationale}

Widal test works on the principle of agglutination and it is the only serological test available to diagnose enteric fever. If the performance of semi quantitative slide widal test in estimating the titres is found satisfactory, then turn around time for diagnosis of enteric fever and start of treatment can be reduced from 24 hours to 10 minutes.

Since slide widal test does not require additional glassware/instruments or involve complex procedure as in tube widal, moderately skilled technician can perform the test in small resource limited settings. This will benefit remote areas of the country where sophisticated lab facilities are absent. Also cost incurred on inventories and performance of widal can be largely reduced.

The main aim of this study, Comparison of performance of qualitative and semiquantitative slide widal test and quantitative tube widal test in diagnosis of enteric fever.

Also the main objectives of this study include, to compare the performance of qualitative slide Widal test against the semi-quantitative tube Widal test. And to compare the performance of semiquantitative slide Widal test against the semi-quantitative tube Widal test.

\section{Inclusion criteria}

In patients and out patients with probable Enteric fever attending various clinical departments of our hospital

Patient presenting with fever for 1 to 2 weeks.

\section{Exclusion criteria}

Chronic enteric fever. 
Recurrent enteric fever.

Recently vaccinated with $T A B$ vaccine.

Patients with Bleeding disorders.

Patient who did not give informed written consent for the study.

Cross infection with Proteus, Rickettesial infection etc...

\section{Materials and Methods}

$A$ Cross sectional study was conducted after obtaining Institutional Human Ethics Committee Clearance (IHEC), and patient's informed written consent was obtained prior to the commencement of the study. A cross sectional study was undertaken from April 2018 to October 2018 and was conducted in the Department of Microbiology, Karpagam Faculty Of Medical Sciences and Research. About 400 serum samples were obtained from the patients with probable Enteric fever, who constituted the study population. Serum samples received in the Serology section of Diagnostic Microbiology Division, Central Service Laboratory of our Hospital were subjected to both qualitative and semi quantitative analysis using slide and tube widal test respectively as per Manufacturer's Instructions (Span Diagnostic Kit).

Serum samples were evaluated for the presence of Genus specific antibodies to poly 'O' Antigen (Salmonella typhi), and also for the presence of species specific antibodies against ' $\mathrm{H}$ ' Antigen (Salmonella typhi), 'AH' Antigen (Salmonella paratyphi A), 'BH' Antigen (Salmonella paratyphi B) respectively. Standard tube widal agglutination test was performed by making serial dilutions of the serum by using isotonic normal saline and $0.5 \mathrm{ml}$ of each antigen suspension $(\mathrm{O}, \mathrm{H}, \mathrm{AH}, \mathrm{BH})$ were added to the corresponding tubes (6 tubes), Comprising different dilutions viz., 1:20, 1:40, 1:80, 1:160, $1: 320,1: 640$. Antigen control to detect Autoagglutination is also done simultaneously $\left(7^{\text {th }}\right.$ tube). Dreyers tube (round bottom) was used for demonstrating antibodies against ' $\mathrm{O}$ ' Antigen and Felix tube (conical bottom) are used to demonstrate antibodies to ' $\mathrm{H}$ ', 'AH', 'BH' antigens respectively.

Then these tubes were incubated at $37^{\circ} \mathrm{C}$ for 24 hours and the reading of the tubes was done on the next day. Tubes showing 50\% agglutination viz., for ' $\mathrm{O}$ ' antigen - purple granular deposits along with clearing of the tube $\geq 2 / 3^{\text {rd }}$ of the suspension was considered positive. And for ' $\mathrm{H}$ ', 'AH', 'BH' antigen cotton wooly clumps along with $\geq 2 / 3^{\text {rd }}$ clearing of the suspension in the tube is considered as positive.

In case of semi-quantitative slide widal and tube widal agglutination test, titres of $\geq 1: 80$ is considered as significant titre for Antibodies to ' $\mathrm{O}$ ' antigen, $\geq 1: 160$ is considered as significant titre for antibodies to ' $\mathrm{H}$ ', ' $\mathrm{AH}$ ', 'BH' antigens respectively. Qualitative widal agglutination tests are done using one drop $(50 \mu 1)$ of patients serum and one drop $(50 \mu 1)$ of desired antigen $(\mathrm{O}, \mathrm{H}, \mathrm{AH}, \mathrm{BH})$. Presence or absence of visible agglutination -granular clumps, at the end of a minute gentle rotatory shaking is interpreted as positive and negative respectively. Quality control was done using positive and negative quality control given along with the kit as per manufacturer's instruction. Four fold rise or decrease in titres between acute phase sera and convalescent phase sera is considered as significant titre for this serological test. And finally the results were tabulated for further statistical analysis using SPSS version 23.0 statistical software.

\section{Results and Discussion}

Out of 400 paired serum samples, Prevalence of Enteric fever by Tube agglutination and slide agglutination test are 23\% (92 out of 400 
cases) and $13 \%$ (52 out of 400 cases) respectively. Incidence of Typhoid fever, paratyphoid ' $A$ ' fever and paratyphoid 'B' fever were found to be $13 \%$ (52 out of 400 cases), 0 ( 0 out of 400 cases) and 0 ( 0 out of 400 cases) respectively by slide widal test. The incidence of Typhoid, paratyphoid ' $A$ ' fever and paratyphoid ' $\mathrm{B}$ ' fever was found to be $23 \%$ (92 out of 400 cases), 0 (0 out of 400 cases), 0 (0 out of 400 cases) respectively by tube widal test. Chi-square test was applied, the results obtained by the Qualitative and Semi quantitative slide and tube widal test was found to be statistically significant. $\left(X^{2}=113.31\right), d f=1(p<0.0001)$

Enteric fever is one of the significant health problems in the community because of its mortality and morbidity, the diagnosis of Enteric fever can be made by testing the patients stool, blood, urine and other body fluids. Among which the Gold standard for diagnosing enteric fever is by isolation of bacteria by culture method but it takes almost a week to obtain the results, so then tube widal is the next best option which takes 24 hours to obtain the result, and recently slide widal was subsequently introduced for diagnosing enteric fever which almost gives results within Minutes. Among 400 samples tested for salmonella species, the sensitivity and specificity of the slide widal test is $80 \%$ and $85 \%$ respectively. Sensitivity and specificity of the tube widal is $84 \%$ and $96 \%$ respectively. Positive and Negative predictive value of the slide and tube widal test was $82 \%$ and $85 \%$ respectively.

Our results Matched the study done elsewhere, Thelma E. Tupasi et al., in their study from various developing countries endemic to typhoid fever, found the sensitivity and specificity of tube Widal ranging from 61$90 \%$ and $88-100 \%$ respectively and that of slide agglutination method to be $53-80 \%$ (sensitivity) and $57-90 \%$ (specificity) (Tupasi et al., 1991). Keddy H Karen et al., in their study concluded that the semiquantitative slide agglutination test had shorter turnaround times than the Widal tube test and poor specificity and hence should not be used as diagnostic tool (Keddy et al., 2011). John Wain et al., stated that the quantitative slide agglutination test used instead of standard Widal test should always be interpreted with reference to clinical data (Wain John et al., 2005).

Endemic titre of Salmonella typhi among healthy population was reported to be 1:40 and 1:80, respectively. And the Endemic titre of Salmonella paratyphi $A$ and $B$ were found to be 1: 40 and 1:80, respectively (Rucha Patki et al., 2017). Although blood culture remains the gold standard test for diagnosing enteric fever, it may not be available in many rural areas. In that case tube widal can be used, which is relatively skill based, time consuming, inexpensive and Non-invasive. And when compared with the slide widal test, sensitivity and specificity of tube widal test is much remarkable than the slide widal test, so it can be indefinitely used as a diagnostic test.

\section{Limitations of the Study}

Time duration of the study is short. Sample size is less to arrive at a definitive conclusion. Cost effectiveness is a factor.

Post-centrifugation Hemolysis (practical issues).

Widal test is used to detect antibodies against Salmonella typhi, Salmonella paratyphi A and $B$ only but no other Salmonella species, which are also human pathogens of medical importance but rare. 
Table.1 Interpretation of Tube widal test

\begin{tabular}{|c|c|c|c|c|}
\hline Interpretation of Tube Widal test & $\mathbf{O}$ & H & $\mathbf{A H}$ & BH \\
\hline $\begin{array}{l}\text { Positive for Salmonella typhi (Typhoid) } \\
\text { fever }\end{array}$ & $\geq 1: 80$ & $\geq 1: 160$ & $\leq 1: 20$ & $\leq 1: 20$ \\
\hline $\begin{array}{l}\text { Positive for Salmonella paratyphi A } \\
\text { (paratyphoid 'A' fever) }\end{array}$ & $\geq 1: 80$ & $\leq 1: 160$ & $\geq 1: 160$ & $<1: 160$ \\
\hline $\begin{array}{l}\text { Positive for Salmonella paratyphi B } \\
\text { (paratyphoid 'B' fever) }\end{array}$ & $\geq 1: 80$ & $\leq 1: 160$ & $<1: 160$ & $\geq 1: 160$ \\
\hline Negative for Enteric fever & $<1: 80$ & $<1: 160$ & $<1: 160$ & $<1: 160$ \\
\hline
\end{tabular}

Table.2 Incidence of Enteric fever

\begin{tabular}{|l|}
\multicolumn{1}{|c|}{ Study population $(\mathrm{N}=400)$} \\
\hline Incidence of Salmonella typhi \\
\hline Incidence of Salmonella paratyphi A \\
\hline Incidence of Salmonella paratyphi B \\
\hline Total number of Enteric fever cases \\
\hline Negative for Enteric fever \\
\hline
\end{tabular}

Anamnestic response due to recent vaccination and cross infection

Prozone phenomenon of antibody excess.

\section{Recommendations}

Hospital antibiotic policy to be adhered and revised periodically. Serological profile of salmonella infection would help us to understand type prevalence and can be a potential tool for Epidemiological study and infection control. Molecular methods like polymerase chain reaction, DNA probes could be the future scope.

Large number of samples can be subjected for the comparison between the slide and tube widal test to make the results still authoritative. Comparison of effectiveness between commercially available different widal kits. Comparison of results of widal test between the patients who have and have not consumed antibiotics prior to the widal test.

\begin{tabular}{|c|c|}
\hline $\begin{array}{c}\text { SLIDE WIDAL } \\
(\mathbf{N}, \%)\end{array}$ & $\begin{array}{c}\text { TUBE WIDAL } \\
(\mathbf{N}, \%)\end{array}$ \\
\hline$(52,13 \%)$ & $(92,23 \%)$ \\
\hline$(0,0)$ & $(0,0)$ \\
\hline$(0,0)$ & $(0,0)$ \\
\hline 52 out of 400 cases & 92 out of 400 cases \\
\hline$(348,87 \%)$ & $(308,77 \%)$ \\
\hline
\end{tabular}

Prospective study can be conducted to find the clinical correlation of the patient, who was positive for widal test.

Prevalence of Enteric fever evaluated by slide and tube widal agglutination tests were found to be $13 \%$ and $23 \%$ respectively. Though blood culture is the Gold standard for diagnosis of Enteric fever, the sensitivity is better only during first week of fever. Especially during second week of fever we could use widal test as an alternative or supplementary test of choice. We conclude our study with the fact that the performance of tube widal is better than the slide widal test.

Hence slide widal test alone cannot be used for diagnosis of Enteric fever especially during second week of fever. Though slide widal test is easy to perform and gives rapid results than the tube widal test, slide widal could only be considered as a supplementary test along with tube widal agglutination test. 
Diagnosis of enteric fever earlier is very important, because of the prescription of irrational antibiotics where the results tends to be negative, so in that case tube widal test plays a major role in diagnosis of enteric fever. But now at present slide widal has been introduced in the diagnosis of the enteric fever. So this research is done to compare the qualitative and semi quantitative results of both the test, of which the results came satisfactory with tube widal which is highly superior in diagnosing the disease when compared to slide widal test, which can be applied as the diagnostic test.

\section{Acknowledgement}

This study was done as a part of Indian Council of Medical Research Short Term Studentship project (ICMR STS 2018). I thank ICMR for accepting my research project. I am thankful to the Dean and HOD of Microbiology for permitting to do the study.

\section{References}

Dandona R, Kumar et al., Nations within a nation: variations in epidemiological transition across the states of India, 1990-2016 in the Global Burden of Disease Study. India State-level Disease
Burden Initiative Collaborators. The Lancet, 2017; 390 (10111): 2437-60.

John J, Van Aart C. J, Grassly N. C. The burden of typhoid and paratyphoid in India: systematic review and metaanalysis. PLoS Negl. Trop.Dis, 2016; 10 (4): e0004616. 10.1371/journal. pntd.0004616.

Keddy $\mathrm{H}$ Karen et al., Sensitivity and Specificity of typhoid fever rapid antibody tests for laboratory diagnosis at two Sub-saharan African sites. Bulletin of WHO, 2011; 89: 640-47.

Mengist HM, and Tilahun K. Diagnostic Value of Widal test in the Diagnosis of Typhoid Fever: A Systematic Review. $J$ Med Microb Diagn, 2017; 6: 248. doi: 10.4172/2161-0703.1000248.

Olopoenia LA, and King AL. Widal agglutination test - 100 years later: Still plagued by controversy. Postgrad Med $J, 2000$; 76: 80-4.

Rucha Patki et al., Baseline antibody titre against Salmonella enterica in Healthy individuals, India. 2017: 9042125.

Tupasi E. Thelma et al., Clinical Application of the widal test. Phil J Microbiol Infect Dis 1991; 20 (1): 23-6.

Wain John et al., The laboratory diagnosis of enteric fever. $J$ infect Developing countries. 2005; 421-25.

\section{How to cite this article:}

Keerthana, N.., R. Someshwaran and Dhanasekaran, A. 2018. Comparative Evaluation of Performance of Qualitative and Semiquantitative Slide Widal Test versus Quantitative Tube Widal Test in Diagnosing Enteric Fever. Int.J.Curr.Microbiol.App.Sci. 7(11): 1714-1719. doi: https://doi.org/10.20546/ijcmas.2018.711.196 